\title{
RETRACTED ARTICLE: Y Chromosomal STR haplotypes in Chinese Uyghur, Kazakh and Hui ethnic groups and genetic features of DYS448 null allele and DYS19 duplicated allele
}

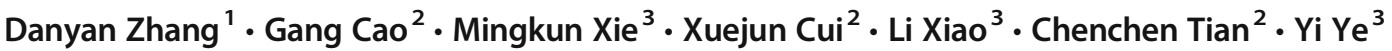

Received: 19 December 2018 / Accepted: 20 March 2019 / Published online: 29 March 2019

(C) Springer-Verlag GmbH Germany, part of Springer Nature 2019

This article [1] has been retracted at the request of the corresponding author, Yi Ye, after concerns were raised regarding the ethics approval and consent procedures. The corresponding author informed the Publisher that contrary to the ethics statement in the article, the study was undertaken without the approval of their institutional ethics committee. As the study fails to meet the ethical standards required by the Journal, it has been retracted.

Yi Ye and Danyan Zhang agree to this retraction. Gang Cao, Mingkun Xie, Xuejun Cui, Li Xiao and Chenchen Tian have not responded to correspondence about this retraction.

\section{Reference}

[1] Zhang, D., Cao, G., Xie, M. et al. Int J Legal Med (2019). https://doi.org/10.1007/s00414-019-02049-6

Electronic supplementary material The online version of this article (https://doi.org/10.1007/s00414-019-02049-6) contains supplementary material, which is available to authorized users.

\section{Yi Ye}

yeyiconan@163.com

1 Chongqing Population and Family Planning Science and Technology Research Institute, Key Laboratory of Birth Defects and Reproductive Health, Chongqing 400020, China

2 Criminal Policeman Detachment, Karamay Municipal Public Security Bureau, Karamay 834000, Xinjiang, China

3 Department of Forensic Toxicological Analysis, West China School of Basic Medical Sciences \& Forensic Medicine, Sichuan University, 8th Floor, Fa Yi Building, No.16, Section 3, Renmin Nan Road, Chengdu 610016, Sichuan, China 